\title{
Bacterial Stimulation of the TLR-MyD88 Pathway Modulates the Homeostatic Expression of Ileal Paneth Cell $\alpha$-Defensins
}

\author{
A. Menendez ${ }^{a} \quad$ B.P. Willing ${ }^{a} \quad$ M. Montero $^{b}$ M. Wlodarska ${ }^{a} \quad$ C.C. So ${ }^{a}$ \\ G. Bhinder $^{\text {b }}$ B.A. Vallance ${ }^{b}$ B.B. Finlay ${ }^{a}$ \\ ${ }^{a}$ Michael Smith Laboratories, The University of British Columbia, and b Division of Gastroenterology, BC's Children's \\ Hospital and Child and Family Research Institute, Vancouver, B.C., Canada
}

\section{Key Words}

Defensins · Toll-like receptors • Intestinal microbiota •

Paneth cells $\cdot$ Antimicrobial peptides $\cdot$ Cryptdins

\begin{abstract}
Paneth cell $\alpha$-defensins are antimicrobial peptides involved in the control of the intestinal microbiota and immunological homeostasis. In mice, they are encoded by multiple, highly homologous genes (Defa). The transcriptional activity of ileal Defa genes was studied in response to pharmacological and genetic perturbations of the intestinal environment of C57BL/6 mice. Defa gene transcription was sensitive to oral antibiotic administration suggesting that commensal microbes regulate Defa expression. Ileal microbiota analysis showed that decreased transcription of Defa genes correlated with depletion of Lactobacillus. Defa expression was partially restored in vivo by lactobacillus administration to antibiotic-treated mice. Defa transcripts were less abundant in ex vivo, microbiota-free intestinal explants but recovered after explant exposure to UV-killed bacteria, Toll-like receptor (TLR)-2 or TLR4 agonists. Genetic deficiency of several TLRs or MyD88 led to dramatic drops in Defa transcription in vivo. These results show that Paneth cell Defa genes are regulated by commensal bacteria through TLR-MyD88 signaling and
\end{abstract}

(C) 2012 S. Karger AG, Basel

1662-811X/13/0051-0039\$38.00/0

ax +4161306 1234

E-Mail karger@karger.ch

www.karger.com
Accessible online at: www.karger.com/jin provide a further understanding of the dysregulation of intestinal homeostasis that occurs as a result of imbalances in the populations of commensal bacteria.

Copyright $\odot 2012$ S. Karger AG, Basel

\section{Introduction}

Together with other antimicrobials, enteric $\alpha$-defensins are secreted into the lumen of the mammalian small intestine [1]. They are thought to defend against pathogenic insults as suggested by in vivo studies, where gain of a human defensin 5 (HD5) in a transgenic mouse model improved protection from enteric Escherichia coli and Salmonella infections whereas deficiency of Mmp7 (the enzyme responsible for processing cryptdin precursors to their mature form) correlated with increased susceptibility to those infections $[2,3]$. In addition, recent evidence indicates that enteric $\alpha$-defensins participate in the control of intestinal homeostasis by modifying the commensal microbial composition and in turn, influencing the immunological landscape of the intestinal mucosa [4].

\section{A.M. and B.P.W. contributed equally to this work.}

Dr. B. Brett Finlay

Michael Smith Laboratories, The University of British Columbia 301-2185 East Mall

Vancouver, BC V6T 1 Z4 (Canada)

Tel. +1 604822 2210, E-Mail bfinlay@interchange.ubc.ca 
In the healthy adult gut, enteric $\alpha$-defensins are produced exclusively by Paneth cells in the crypts of the small intestine [1]. In mice, enteric $\alpha$-defensins, the cryptdins, are encoded by a group of highly homologous genes (Defa), located on the defensin gene cluster on chromosome 8 . The repertoire of $\mathrm{C} 57 \mathrm{BL} / 6$ mice includes more than 20 potentially active Defa genes and numerous pseudogenes $[5,6]$. In addition, the C57BL/6 Defa genotype is different from that of other laboratory mouse strains; more than 10 transcriptionally active genes identified in other mouse lines have not been found in C57BL/6 and several genes are unique to this mouse strain $[5,6]$.

Expression analyses of Defa genes have been of limited use in understanding $\alpha$-defensin biology in mouse models of enteric diseases. A major problem is the high gene sequence homology, which makes PCR primer design challenging and clouds the validity of microarray data. This fact, combined with the incomplete knowledge of the strain diversity in the Defa gene pool, has led to the generation of a significant amount of data that require reinterpretation in light of the recent C57BL/6 Defa genotyping $[5,6]$. Other than an involvement of the $\beta$-catenin pathway $[7,8]$, very little is known about the control of intestinal $\alpha$-defensin expression. Previous studies on the role of bacteria in cryptdin synthesis have produced conflicting results [9-12]. Here, we studied the transcriptional activity and regulation of ileal Defa genes in adult C57BL/6 mice by qPCR. Two major, unique aspects of this work are the generation and validation of primers specific for the Defa gene repertoire of this mouse strain, and the analysis of the majority of the known genes of C57BL/6 mice. We found significant variation in the steady-state Defa gene expression, which was responsive to antibiotic-induced perturbations of the intestinal environment. Most interestingly, Defa transcriptional activity correlated to specific changes in the composition of the intestinal microbiota and was affected by genetic deficiencies in the TLR-MyD88 signaling pathway. Collectively, these findings show that Paneth cell Defa gene expression is controlled by the intestinal commensal microbiota through the TLR-MyD88 pathway.

\section{Materials and Methods}

\section{Animals}

Eight- to twelve-week-old, wild-type C57BL/6 and congenic sex- and age-matched MyD88-/-, Tlr2-/-, Tlr4-/-, Tlr2-/-/ Tlr4-/-, Tlr9-/- or Nod2-/- mice were raised in the same room in the animal facility of the Child and Family Research Institute. All knockout mouse strains were backcrossed onto a C57BL/6 ge- netic background between 6 and 10 times and housed in the same room as wild-type C57BL/ 6 for a minimum of 3 years. Animal protocols for this study were approved by the University of British Columbia's Animal Care Committee. Antibiotics were administered in the drinking water for 4 days at $450 \mathrm{mg} / \mathrm{l}$ streptomycin or $750 \mathrm{mg} / \mathrm{l}$ metronidazole. Mice were sacrificed and tissue samples of the terminal ileum were taken approximately 1 inch from the ileocecal junction for RNA purification, analysis of microbiota and microscopy.

\section{Ileal Explants}

Ileal explants were prepared essentially as described [13]. Briefly, the distal region of the ileum was resected and flushed with sterile PBS. The tissue was opened longitudinally and washed repeatedly with sterile $\mathrm{PBS}$ to eliminate the remaining intestinal content and immediately placed in DMEM/10\% FCS $/ 100 \mu \mathrm{g} / \mathrm{ml}$ streptomycin $/ 100 \mathrm{U} / \mathrm{ml}$ penicillin. The tissues were divided into 2-mm-long sections in sterile conditions and incubated at $37^{\circ} \mathrm{C}$ in humidified $5 \% \mathrm{CO}_{2}$ atmosphere. After $1 \mathrm{~h}$, the media were replaced and the samples were incubated for an additional $2 \mathrm{~h}$ in the same medium. Thereafter, the explants were transferred to fresh media containing E. coli lipopolysaccharide (LPS) (Sigma), Pam2CSK4, Pam3CSK4 (InvivoGene, http://www.invivogen. com/, cat. No. tlrl-pm2s and tlrl-pms) or UV-killed Lactobacillus johnsonii, Lactobacillus reuteri or E. coli (all previously isolated from the intestinal commensal population of experimental animals) and incubated for $16 \mathrm{~h}$ at $37^{\circ} \mathrm{C}$ in a humidified $5 \% \mathrm{CO}_{2}$ atmosphere.

\section{Expression Analysis}

Sections and ileal explants were collected in RNA Later (Qiagen) and total RNA was isolated using the RNeasy kit (Qiagen). cDNA was prepared from $1 \mu \mathrm{g}$ of RNA using the Quantitech Reverse Transcription kit (Qiagen). Quantitative PCRs (qPCRs) were done on an Applied Biosystems 7500 using the DyNamo SYBR Green PCR Kit from New England Biolabs. The qPCR primers and conditions for the Defa genes are shown in table 1, the primers for the housekeeping gene 36B4 [ribosomal protein, large, P0 (Rplp0), NM_007475.5] were TCTGGAGGGTGTCCGCAAC (forward) and CTTGACCTTTTCAGTAAGTGG (reverse); the primers for matrix metalloproteinase $7(\mathrm{Mmp} 7)$ were CACTCTAGGTCATGCCTTCGC (forward) and GGTGGCAGCAAACAGGAAGTTC (reverse). Efficiencies for the 36B4 PCRs were 1.9055 at $58^{\circ} \mathrm{C}, 1.9333$ at $60^{\circ} \mathrm{C}$ and 1.8544 at $62^{\circ} \mathrm{C}$. qPCR specificity was verified by cloning of PCR products using TOPO TA 4.0 vector and $E$. coli TOP 10 chemically competent cells (Invitrogen, Carlsbad, Calif., USA) and sequencing of 10-20 independent clones. Relative expression data were generated using the ddCt method, corrected for primer efficiencies according to Pfaffl et al. [14]. Relative expressions from intestinal explants were normalized against the relative expression of villin (expressed exclusively in the epithelial cell layer) as an indication of epithelial survival. The data were normalized for each sample using the formula Rel $\operatorname{Exp}_{\text {(gene of interest) }} / \operatorname{Rel} \operatorname{Exp}_{\text {(villin). }}$.

\section{Microbial Analysis}

Total bacteria were enumerated by SYBR green DNA staining. Ileal contents were homogenized in $1 \mathrm{ml}$ of PBS and then fixed in $3.7 \%$ formalin. Fixed samples were diluted and applied to $0.2 \mu \mathrm{m}$ Anodisc 25 filters (Whatman International Ltd.), then stained 
Table 1. The gene composition of each amplification group as defined in this work

\begin{tabular}{|c|c|c|}
\hline Genes included in group & Group name & Remarks \\
\hline Defa3 and OTTMUSG00000019892 & Defa3 & Gene duplication, identical genes and protein products \\
\hline Defa5-related genes* & Defa5 & $\begin{array}{l}\text { Four genes differing in one or two nucleotides in the pre- or pro- re- } \\
\text { gions but producing identical mature proteins, identical PCR products }\end{array}$ \\
\hline Defa $20^{* *}$ and OTTMUSG00000019860 & Defa20 & Gene duplication, identical genes and protein products \\
\hline Defa 21 and Defa22 & Defa21/22 & Two different genes, amplified together, different PCR products \\
\hline Defa23 and OTTMUSG00000019762 & Defa23 & Gene duplication, identical genes and protein products \\
\hline Defa25 and OTTMUSG00000019857 & Defa25 & TATA box-less promoters \\
\hline Defa26 and OTTMUSG00000019784 & Defa26 & Two different genes, identical mature proteins, identical PCR products \\
\hline Defa4 & Defa4 & Gene not present in C57BL/6 mice \\
\hline
\end{tabular}

With the exception of Defa4, gene ID numbers are from the Vertebrate Genome Annotation (VEGA) database (http://vega. sanger.ac.uk/index.html) following Amid et al. [5].

The VEGA database ID numbers are Defa3 (OTTMUSG00000019782), Defa20 (OTTMUSG00000019856), Defa21 (OTTMUSG00000019489), Defa22 (OTTMUSG00000019763), Defa23 (OTTMUSG00000019488), Defa24 (OTTMUSG00000019980), Defa25 (OTTMUSG00000019700) and Defa26 (OTTMUSG00000019889). Defa4 is NCBI NM_010039.
* Defa5-related genes include OTTMUSG00000018258, OTTMUSG00000019785, OTTMUSG00000018259 and OTTMUSG00000019924.

** The names of the Defa genes as used here correspond to and substitute former designations such as Cryp (e.g. Defa 3 is Cr3 or Cryp3, or Defcr3) and so on, with the exception of Defa20, which has been also called Cr4-C57B6 or Crp4 (B6a) [10, 34] and Defa21/22, which have been called Crp4 (B6b) [34]. with SYBR green (Invitrogen), diluted 1:400 in PBS, for 15 min. Filters were mounted on glass slides with $30 \mu \mathrm{l}$ of antifade solution. Images were collected on an Olympus IX81 microscope from 3 randomly chosen fields for each sample and cell counts were averaged. Microbial composition was assessed in tissue samples collected from the ileum by terminal restriction fragment length polymorphism analysis (T-RFLP) and cloning and sequencing. Total DNA was isolated from ileal tissue using the QIAamp DNA mini kit (Qiagen) according to the manufacturer's instructions, with the addition of a 60-second bead-beating step at level 5 on a FastPrep-24 (MP Biomedicals, Solon, Ohio, USA). Bacterial 16S rRNA genes were PCR amplified using broad-range primers $8 \mathrm{~F}$ [15] 5'-end-labeled with 6-FAM, and 926r [16] and subjected to digestion with HaeIII. Fragment length determination of 6-FAMlabeled products was performed on an ABI 3730 capillary sequencer (Applied Biosystems) and electrophoregrams were processed using GeneMarker (State College, Pa., USA). For the phylogenetic identification of terminal restriction fragments of interest, pooled PCR-amplified products from each treatment group were cloned into the $\mathrm{pCR}{ }^{\circledR} 4$ TOPO vector and transformed into E. coli TOP10 chemically competent cells (Invitrogen) and 64 clones per treatment were sequenced. Sequences were classified using the naïve Bayesian rRNA classifier in Ribosomal Database Project [17]. Selective culture of lactic acid bacteria was performed on Rogosa agar under anaerobic conditions.

\section{Microscopy}

Tissue sections were prepared and stained with hematoxylineosin (HE) by WaxIt Inc. (Vancouver, B.C., Canada). HE images were taken in an Olympus VS110 using the VS-ASW software. Immunofluorescent $\mathrm{Ab}$ localization was performed on paraffin- embedded tissue sections ( $5 \mu \mathrm{m}$ ) after paraffin removal with xylene and rehydration by sequential immersion in 100, 95 , and $75 \%$ ethanol and water. Antigen retrieval was performed in $10 \mathrm{mM}$ citric acid $\mathrm{pH} 6.0$ at $100^{\circ} \mathrm{C}$ for $30 \mathrm{~min}$. Immunostaining was performed using a rabbit anti-human lysozyme (Dako A0099, 1:600). Fluorescence was visualized using a Zeiss AxioImager 2 and processed with the AxioImager software.

\section{Data Analysis}

Data were analyzed with GraphPad Prism version 4.0 (GraphPad Software Inc., San Diego, Calif., USA). Statistical comparisons were done with Mann-Whitney A tests with 95\% confidence intervals. Sequence alignment and the phylogenetic tree were done using ClustalW2 (http://www.ebi.ac.uk/Tools/msa/clustalw2/).

\section{Results}

Differential Expression of Defa Genes in the Terminal Ileum of C57BL/6 Mice

A careful analysis of the published work on cryptdin gene expression reveals that (1) frequently, primer sets designed to amplify one particular Defa gene are potentially able to cross-amplify others; this problem is often aggravated by failure to report the PCR conditions and lack of product sequence verification, (2) in vivo expression data for genes not yet found in C57BL/6 mice are 
Table 2. The primers set for the C57BL/6 Defa genes qPCR

\begin{tabular}{|c|c|c|c|c|}
\hline Gene group & Primers sequences & $\begin{array}{l}\text { Product } \\
\text { length, bp }\end{array}$ & $\begin{array}{l}\mathrm{qPCR} \\
\text { annealing, }{ }^{\circ} \mathrm{C}\end{array}$ & $\begin{array}{l}\text { qPCR } \\
\text { efficiency }\end{array}$ \\
\hline Defa3 & $\begin{array}{l}\text { F: GAGAGATCTGGTATGCTATTG } \\
\text { R: AGCAGAGTGTGTACATTAAATG }\end{array}$ & 98 & 62 & 1.956 \\
\hline Defa5 & $\begin{array}{l}\text { F: TCAAAAAAGCTGATATGCTATTG } \\
\text { R: AGCTACAGCAGAATACGAAAG }\end{array}$ & 106 & 58 & 1.982 \\
\hline Defa20 & $\begin{array}{l}\text { F: GAGAGATCTGGTATGCTATTG } \\
\text { R: AGAACAAAAGTCGTCCTGAG }\end{array}$ & 86 & 62 & 2.125 \\
\hline Defa $21 / 22$ & $\begin{array}{l}\text { F: GAGAGATCTGATCTGCCTTTG } \\
\text { R: CAGCGCAAAAAAGGTCCTGC }\end{array}$ & 87 & 64 & 2.079 \\
\hline Defa23 & $\begin{array}{l}\text { F: GAGAGATCTGGTATGCTATTG } \\
\text { R: AGCAGAGCGTGTATATTAAATG }\end{array}$ & 98 & 62 & 1.376 \\
\hline Defa24 & $\begin{array}{l}\text { F: GAGAGATCTGGTATGCTATTG } \\
\text { R: AGCAGAGCATGTACAATAAATG }\end{array}$ & 98 & 62 & 1.556 \\
\hline Defa 25 & $\begin{array}{l}\text { F: GTGAAGATCTGATATGCTATTG } \\
\text { R: ACCAGAGCATGTACATTAAATG }\end{array}$ & 99 & 58 & 1.980 \\
\hline Defa26 & $\begin{array}{l}\text { F: ATTGTAGAAAAAGAGGCTGTAC } \\
\text { R: AGCAGAGTGTGTACATTAAATG }\end{array}$ & 81 & 62 & 1.802 \\
\hline Defa $4^{*}$ & $\begin{array}{l}\text { F: TGAGAGGTTTGTTATGCTATTG } \\
\text { R: AGCAGTACAAAAATCGTATTCC }\end{array}$ & 90 & 60 & 1.992 \\
\hline
\end{tabular}

Reaction cycling conditions were: one enzyme activation step at $95^{\circ} \mathrm{C}$ for $10 \mathrm{~min}$ followed by 40 cycles of $95^{\circ} \mathrm{C}, 30 \mathrm{~s}$, annealing for $30 \mathrm{~s}$ at the specified temperature and extension at $72^{\circ} \mathrm{C}$ for another $30 \mathrm{~s}$. Fluorescence readings were taken during the extension time.

* Not present in C57BL/6 mice.

often provided in studies using this mouse strain (e.g. Defa1, Defa 4 and Defa10), probably as a result of the primer design problem mentioned above, (3) in most studies, only one or two Defa genes are analyzed; however, generalized conclusions about Defa expression are made. To avoid these inconsistencies, we designed a novel set of primers for the detection of relative amounts of multiple Defa transcripts by qPCR. The high homology of the Defa genes prevented the generation of individual primer pairs; instead, the genes were clustered in subgroups based on coding sequence homology and phylogenetic distance of their promoters (online suppl. fig. 1; for all online supplementary material see www. karger.com/doi/10.1159/000341630). Six subgroups were formed including two genes each, coding for identical or quasi-identical mature protein products (table 2). An additional subgroup was created with the four Defa5-related genes and another one with Defa4, a gene cloned from $129 \mathrm{SvJ}$ mice, which has not yet been found in C57BL/6 mice. PCR conditions for specific amplification (table 1) were carefully developed, and were validated by sequencing of cloned PCR products generated with each primer mix. Specific amplification of expected targets was obtained and verified for all primer mixes. Expression of Defa3, Defa5, Defa20, Defa21, Defa22, Defa23, Defa24 and Defa26 was confirmed in C57BL/6 mice (fig. 1) whereas transcripts for Defa 25 were undetectable, consistent with the absence of a TATA box in its promoter [5]. Defa 4 transcripts were not detected in C57BL/6 but were readily detected in BalbC and 129SvJ mice (online suppl. fig. 2). The results in figure 1 show that the homeostatic transcript levels of independent Defa genes are dissimilar in vivo.

\section{Oral Antibiotic Treatments Modify the Defa}

Expression Pattern

Clinical use of antibiotics is frequently associated with the expansion of opportunistic pathogens, such as vanco- 


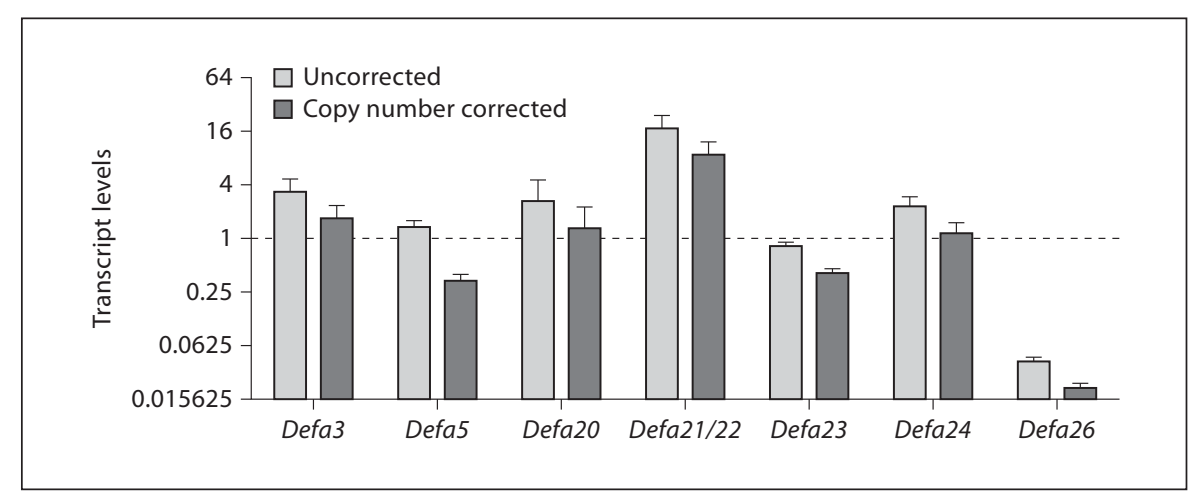

Fig. 1. Paneth cell Defa genes are not equally expressed. Ileal transcript levels of Defa genes in C57BL/6 mice, relative to the transcript levels of the Rplp0 gene (defined as 1, dashed line). Open bars represent the overall relative expression for each subgroup. Solid bars represent relative expression of each individual gene within a subgroup (this was arbitrarily calculated by dividing the overall relative expression of the subgroup by the number of genes within the subgroup; this correction assumes equal expression between the members of each independent subgroup; 2 genes for Defa3, 20,21/22, 23, 24 and 26, and 4 genes within the Defa5 group). Data shown are medians and ranges from the average of two qPCRs; $\mathrm{n}=5$ animals per group.
Fig. 2. Transcript levels of Defa and $M m p 7$ genes in the ileum of antibiotic-treated mice, relative to untreated animals. The graph shows medians and ranges, the table shows the Mann-Whitney test $\mathrm{p}$ values with respect to the untreated controls; ${ }^{*} \mathrm{p}<$ 0.05: significant differences. Results shown are one representative experiment of three; $\mathrm{n}=4$ females per group.

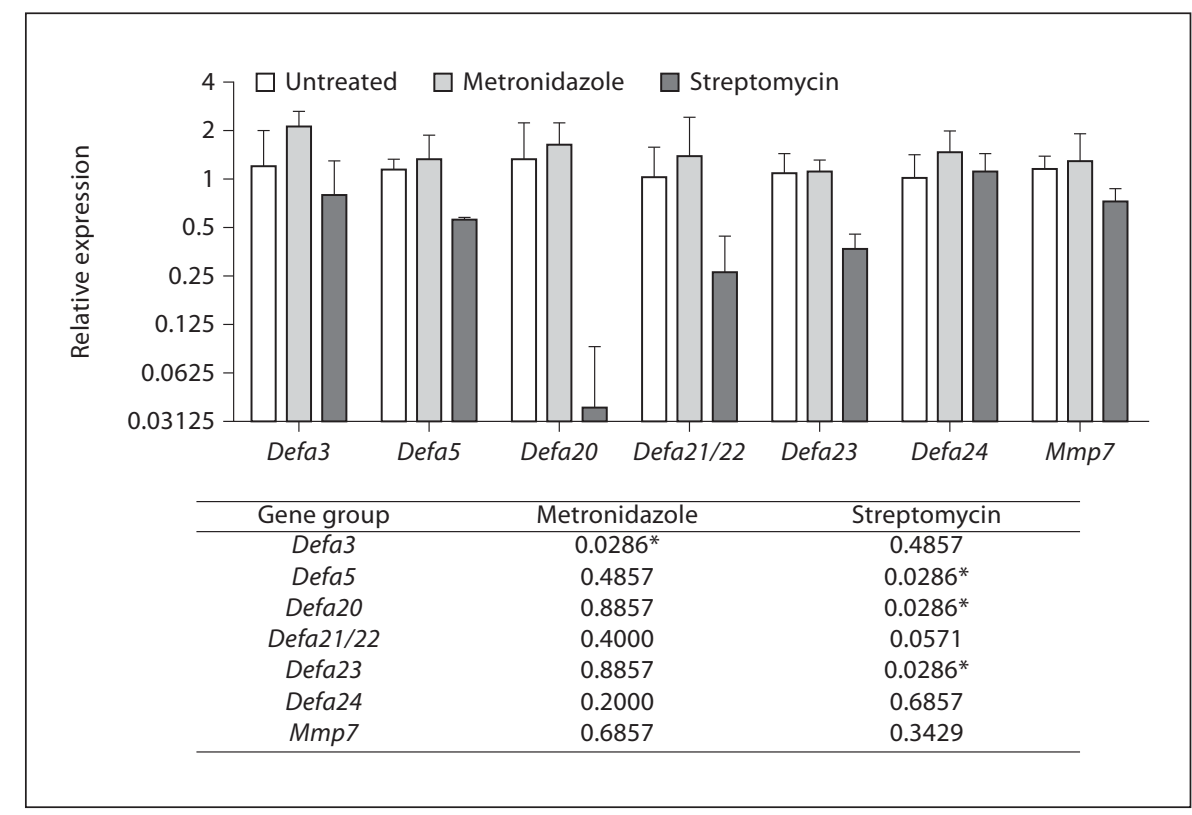

mycin-resistant enterococcus [18] and Clostridium difficile [19]. This is thought to result from alterations of the commensal intestinal microbiota and perturbations of mucosal immune function such as disrupted mucus layer and imbalances in immune cell populations [reviewed in 20]. To determine the susceptibility of Defa expression to antibiotic treatment, independent groups of mice from the same supplier (Jackson) and same housing facility were administered streptomycin or metronidazole, which are known to target distinct bacterial groups (aerobes and anaerobes, respectively). Oral streptomycin treatment for 4 days resulted in decreased expression of several Defa genes (fig. 2) whereas metronidazole induced moderate expression increases that reached statistical significance only for Defa3 (fig. 2). Expression of Mmp7 (which in intestinal epithelial cells is stimulated by bacteria [21]) was unaffected in response to antibiotic administration (fig. 2). Interruption of streptomycin treatment led to a gradual increase in the Defa gene transcripts that were the most clearly affected by treatment (online suppl. fig. 3 ). 




Fig. 3. Changes in microbial populations associated with antibiotic treatment. a Average HaeIII-digested T-RFLP profiles of ileal 16S rRNA gene populations of mice untreated, treated with metronidazole or streptomycin, and 2 or 4 days after suspension of streptomycin treatment. Each bacterial phylotype/TRF peak is represented by a different color; peaks of major interest are marked by hatched bars (TRF232 red and TRF329 blue). ${ }^{*} \mathrm{p}<0.05$ : significant differences. b Total (SYBR staining) and culturable (Rogosa agar) bacteria in ileal contents, data are means $\pm \mathrm{SE}$.

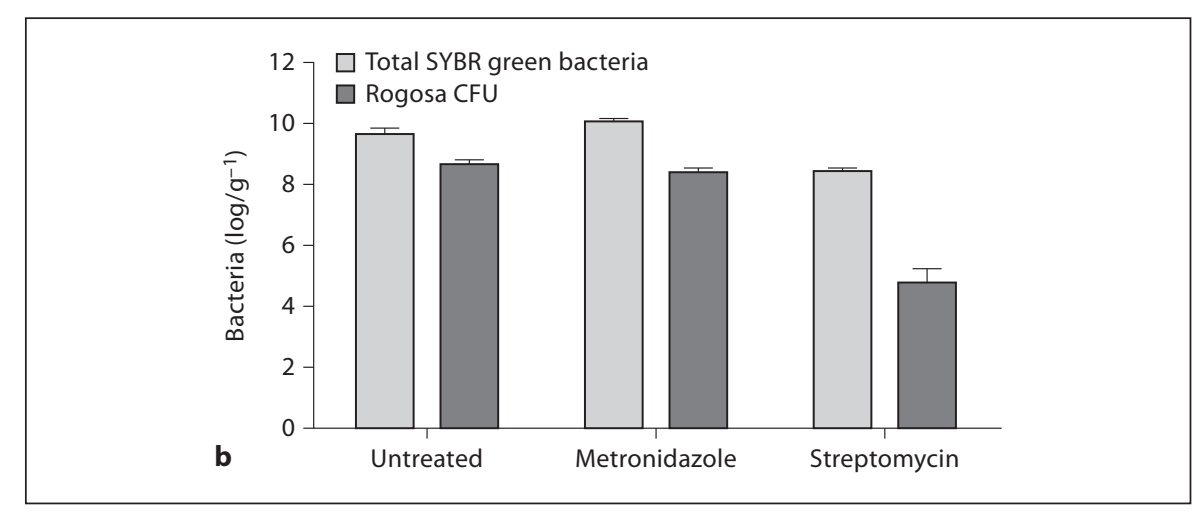

To identify changes in bacterial groups that correlated with changes in Defa expression, the ileal microbial populations of untreated and antibiotic-treated mice were quantified and characterized by T-RFLP and cloning/sequencing of $16 \mathrm{~S}$ rRNA genes (fig. 3a). Streptomycin reduced total luminal bacteria in the ileum (fig. 3b) whereas metronidazole depleted specific bacterial populations. Based on the respective effects of these antibiotics on Defa expression, we aimed to identify candidate microbes that were depleted by streptomycin, unaffected by metro- nidazole and that recovered after streptomycin removal. Only two TRFs, TRF232 and TRF329, fit these criteria. Clone libraries revealed that TRF232 represents a yet uncultured Turicibacter (accession No. EF406422) whereas TRF329 represents members of the genus Lactobacillus. The clone library generated from metronidazole-treated mice was nearly exclusively represented by sequences $>99 \%$ identical to Lactobacillus johnsonii and Lactobacillus reuteri. Changes in the lactobacillus populations of streptomycin-treated mice were confirmed by plating/ 
Fig. 4. a Oral administration of heat-killed commensal bacteria to streptomycintreated wild-type C57BL/6 mice partially restores ileal Defa expression in vivo; the data shown are Defa transcript levels relative to untreated animals. The table shows the Mann-Whitney test $p$ values with respect to the streptomycin-treated animals; ${ }^{*} \mathrm{p}<0.05$ : significant differences. b Exposure to commensal bacteria partially restores Defa expression in ileal explants from wild-type C57BL/6 mice. Results shown are relative Defa expressions normalized to relative villin expression for each sample (RelExp Defa/RelExp villin), as a correction for epithelial survival of the individual explants. The graphs show medians and ranges. Results shown are one representative experiment of two; $n=4$.



isolation on Rogosa agar (fig. 3b) and identification by sequencing of the $16 \mathrm{~S}$ rRNA gene. These results show that the presence of lactobacilli associates with homeostatic Defa transcriptional activity whereas lactobacillus depletion by antibiotic treatment correlates with a drop in ileal Defa gene expression.

The contribution of lactobacilli to the regulation of Defa expression was confirmed by introducing heatkilled $L$. johnsonii and L. reuteri to the drinking water of streptomycin-treated mice. The introduction of $10^{8}$ lactobacilli resulted in a partial rebound in the expression of Defa5, Defa20 and Defa23 whereas a commensal heatkilled E. coli did not have the same effect (fig. 4a). Intriguingly, the expression of Defa3 and Defa24, which reacted rather poorly to streptomycin administration, was significantly reduced by the administration of the heatkilled commensal E. coli. Since the in vivo antibiotic treatments did not completely eliminate the ileal micro- biota, we decided to test the effect of lactobacilli and $E$. coli in cultured intestinal explants. In spite of the limitations inherent in tissue explant culture (such as progressive dying), ileal explants are efficiently devoid of commensals and therefore useful to study their effects on epithelial function. The explants were exposed to UV-killed bacteria for $16 \mathrm{~h}$ and Defa expression measured by qPCR. As shown in figure $4 \mathrm{~b}$, exposure to bacteria induced the transcription of Defa genes, demonstrating that in this model, bacterial stimulation participates in the control of Defa gene expression.

\section{Homeostatic Defa Expression Requires TLR-MyD88 Signaling}

Mice with genetic deficiencies in bacterial sensing (Tlr2-/-, Tlr4-/-, Tlr9-/-, Tlr2/4-/- or MyD88-/-) and C57BL/6 wild-type controls were tested for ileal transcript levels of Defa (all the animals came from colonies 




Fig. 5. TLR2, TLR4 and MyD88 deficiencies cause a drop in the ileal transcript levels of Defa genes. The graph shows medians and ranges; the table shows the Mann-Whitney test $\mathrm{p}$ values with respect to the C57BL/6 wildtype (wt) animals; ${ }^{*} \mathrm{p}<0.05$ and ${ }^{*} \mathrm{p}<0.01$ : significant differences. Results shown are the average of two qPCRs, $\mathrm{n}=6-9$ males per group.

housed at the CFRI). As shown in figure 5, mild to significant reductions in expression were observed in Tlr2-/- mice, most notably for Defa20 and Defa23. Tlr4-/mice showed a more moderate impact, with significant changes only for Defa 3 and Defa 24 although reductions were also observed for other genes. Defa 3 and Defa20 transcription was affected in Tlr9-/- animals, but the rest of the genes were insensitive to TLR9 deficiency. However, the Tlr2-/-/Tlr4-/- double-deficient mice displayed very dramatic reductions in transcript levels for most genes. Deficiency of the downstream adaptor molecule MyD88 also resulted in marked decreases in Defa transcription (fig. 5), in a manner not associated with either gross histological alterations of the intestinal epithelium, reduced number of Paneth cells or reduced levels of lysozyme transcripts (online suppl. fig. 4). Moreover, the expression of Defa 20 was measured in a small group of litermates, co-housed MyD88-/- $(\mathrm{n}=3)$ and $M y D 88-/+$ heterozygous $(\mathrm{n}=2)$ mice derived from heterozygous parents to ensure that differences in expression were not a result of separate housing. The observed differences in transcript levels were consistent with mice housed independently, suggesting that disparities in Defa expression are associated with genotype rather than environment (not shown). In general, transcript levels of Defa in MyD88-/- mice were very similar to those of the Tlr2-/-I Tlr4-/- double knockouts, indicating that the TLRMyD88 pathway is important for homeostatic expression of Defa genes (fig. 5).

In contrast to TLR deficiency, lack of NOD2 had no significant effect on Defa expression, as compared to sexand age-matched C57BL/6 wild-type controls from the same supplier and housed in the same facility (online suppl. fig. 5). Different from Defa genes, Reg3 $\beta$ and Reg $3 \gamma$ expression was insensitive to TLR deficiencies although it was decreased in MyD88-/- mice (online suppl. fig. 6). Our results suggest that TLR2- and TLR4-mediated signaling is a fundamental mechanism for the maintenance of homeostatic expression of Defa genes in the intestinal epithelium of adult mice.

The involvement of TLR2 and TLR4 signaling in Defa expression was verified ex vivo using ileal explants. Ileal 


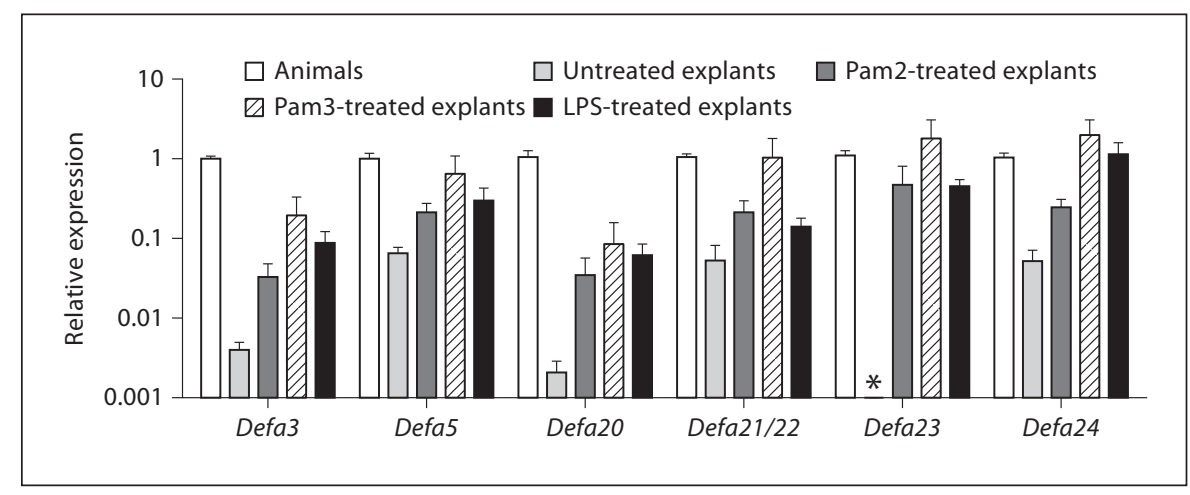

Fig. 6. Treatment with TLR2 and TLR4 agonists (Pam2CSK4, 10 $\mathrm{ng} / \mathrm{ml}$; Pam3CSK4; $100 \mathrm{ng} / \mathrm{ml}$ and LPS, $10 \mathrm{ng} / \mathrm{ml}$ ) restores Defa expression in ex vivo ileal explants from wild-type C57BL/6 mice. Results shown are relative Defa expressions normalized to relative villin expression for each sample (Rel Exp Defa/Rel Exp villin), as a correction for epithelial survival of the individual explants. The asterisk marks the lack of detectable Defa23 transcripts in the untreated explants. Results shown are one representative experiment of two; $\mathrm{n}=4$ or 5 explants per group. Pam2CSK 4 and Pam3CSK 4 are synthetic diacylated and triacylated lipopeptide agonists of the TLR2/6 and 2/1 heterodimers, respectively.

tion. Our results strongly associate Lactobacillus with maximal Paneth cell $\alpha$-defensin expression and show that the commensal population plays a significant role in driving ileal Defa expression. Although it was not tested here, it is quite plausible that other Gram-positive bacteria are also able to induce expression. Observations in clinical specimens from patients with Crohn's disease support the role of commensal bacteria in regulating $\alpha$ defensin expression since both, alterations in the intestinal microbial composition (including the depletion of lactobacilli) and reduction of $\alpha$-defensin levels are observed in patients with Crohn's disease [25, 26].

Antibiotic treatments often lead to secondary clinical intestinal complications $[18,20]$; it is possible that those arise due (at least in part) to alterations of $\alpha$-defensin synthesis profile. There is evidence that Paneth cell $\alpha$-defensins play a role in modulating the intestinal mucosal immune response directly $[27,28]$ and indirectly [4], and have been shown to inhibit the toxin B of C. difficile [29], a common opportunistic pathogen associated with antibiotic therapy [19]. Thus, reductions in Defa expression have the potential to impact the immunological tone of the gut in a significant way.

In contradiction with previous reports [12], we failed to observe any defect of Defa expression in NOD2-deficient mice. In contrast, the TLR-MyD88 pathway seems important for maximal Defa expression. Out of the TLRs tested, TLR2 was the most critical, being important for expression of most gene subgroups. This is in contrast with the work of Dessein et al. [30], who reported that 
TLR2 and MyD88 deficiency had no impact on the expression of cryptdins. However, they studied Defa1 and Defa4, two genes that are absent in C57BL/6 mice. In our study, most Defa genes displayed milder expression reductions in single Tlr2-/- or Tlr4-/- mice than in the double Tlr2-/-/Tlr4-/- or the MyD88-/- strains, suggesting a compensatory action between these two TLRs. This is also supported by the responses of the intestinal explants to both TLR2 and TLR4 agonists.

Activation of the $\beta$-catenin pathway is so far the only mechanism known to regulate Defa gene expression [7, 8]. Our results demonstrate a role of MyD88 for cryptdins expression in the adult mouse intestine, as previously proposed [31] and provide clear evidence of the involvement of at least TLR2 and TLR4. This expands the known function of MyD88 in driving the expression of Paneth cell antimicrobials [32] and highlights the critical role of the TLR-MyD88 pathway in intestinal homeostasis. The normal expression of lysozyme in TLR- and MyD88-deficient mice suggests that the observed defects in Defa expression are not the result of abnormal Paneth cell development.

It is still unclear how the signaling pathway relevant for Defa expression proceeds downstream of MyD88. We observed that Defa expression was insensitive to $\mathrm{NF}_{\kappa} \mathrm{B}_{\mathrm{p} 50}$ null deficiency in vivo (not shown) but considering the functional compensation between the Rel family members [33], these results are not conclusive. It should be noted that all the knockout mice tested here are lacking their specified genes in their germ line and are not cellspecific deficient mice. Thus, the dramatic impact of MyD88 deficiency may not reflect the loss of MyD88 signaling within the Paneth cells, but it may instead reflect indirect actions, i.e. the involvement of MyD88 within other cell types, such as macrophages or myofibroblasts resulting in the release of factors that regulate defensin expression by Paneth cells.
In summary, we have presented here novel observations on ileal Defa gene expression in C57BL/6 mice. Future studies are necessary to establish how the observed differences in transcript levels translate into differences in luminal concentrations of active $\alpha$-defensins and/or intestinal antibacterial activity. We have shown that intestinal commensals are directly linked with $\alpha$-defensin expression through the TLR-MyD88 pathway. It would be interesting to assess whether the synthesis of a related group of antimicrobial peptides, the cryptdin-related sequences, is subject to similar regulation. As alterations of the intestinal microbial flora and homeostasis are associated with an increasing number of pathological conditions (e.g. inflammatory bowel disease, infection and cancer) our findings bear relevance to the understanding of the molecular mechanisms underlying such conditions.

\section{Acknowledgements}

We would like to thank Rémi Gaudreau for assistance with bright-field microscopy. This work was supported by operating grants to B.B.F. from the Canadian Institutes of Health Research (CIHR) and the Crohn's and Colitis Foundation of Canada (CCFC) to B.B.F. and B.A.V. B.B.F. is the UBC Peter Wall Distinguished Professor and B.A.V. is the Canada Research Chair in Pediatric Gastroenterology (Tier 2) and the Children with Intestinal and Liver Disorders (CHILD) Foundation Research Chair in Pediatric IBD Research. A.M. was a recipient of postdoctoral fellowships from the Michael Smith Foundation for Health Research/Genome BC, and the Natural Sciences and Engineering Research Council of Canada. B.P.W. is a recipient of a fellowship from CIHR in partnership with the Institute of Nutrition, Metabolism and Diabetes; the CIHR Strategy on Patient-Oriented Research; and the Canadian Association of Gastroenterology and its partners, the Crohn's and Colitis Foundation of Canada, Abbott, Ferring Pharmaceuticals, and Merck. M.M. is a recipient of postdoctoral fellowships from the Canadian Association of Gastroenterology/CIHR/CCFC and the Child and Family Research Institute. Present address of A.M.: Department of Microbiology and Infectious Diseases, University of Sherbrooke, Sherbrooke, Qué., Canada.

\section{References}

1 Porter EM, Bevins CL, Ghosh D, Ganz T: The multifaceted Paneth cell. Cell Mol Life Sci 2002;59:156-170.

-2 Salzman NH, Ghosh D, Huttner KM, Paterson Y, Bevins CL: Protection against enteric salmonellosis in transgenic mice expressing a human intestinal defensin. Nature 2003; 422:522-526.

-3 Wilson CL, Ouellette AJ, Satchell DP, Ayabe T, Lopez-Boado YS, Stratman JL, Hultgren SJ, Matrisian LM, Parks WC: Regulation of intestinal alpha-defensin activation by the metalloproteinase matrilysin in innate host defense. Science 1999;286:113-117.

-4 Salzman NH, Hung K, Haribhai D, Chu H, Karlsson-Sjoberg J, Amir E, Teggatz P, Barman M, Hayward M, Eastwood D, Stoel M, Zhou Y, Sodergren E, Weinstock GM, Bevins CL, Williams CB, Bos NA: Enteric defensins are essential regulators of intestinal microbial ecology. Nat Immunol 2010;11:7683.
5 Amid C, Rehaume LM, Brown KL, Gilbert JG, Dougan G, Hancock RE, Harrow JL: Manual annotation and analysis of the defensin gene cluster in the $\mathrm{c} 57 \mathrm{bl} / 6 \mathrm{j}$ mouse reference genome. BMC Genomics 2009;10: 606.

6 Shanahan MT, Tanabe H, Ouellette AJ: Strainspecific polymorphisms in Paneth cell alphadefensins of C57BL/6 mice and evidence of vestigial myeloid alpha-defensin pseudogenes. Infect Immun 2011;79:459-473. 
7 van Es JH, Jay P, Gregorieff A, van Gijn ME, Jonkheer S, Hatzis P, Thiele A, van den Born M, Begthel H, Brabletz T, Taketo MM, Clevers $\mathrm{H}$ : Wnt signalling induces maturation of Paneth cells in intestinal crypts. Nat Cell Biol 2005; 7:381-386.

8 Andreu P, Colnot S, Godard C, Gad S, Chafey P, Niwa-Kawakita M, Laurent-Puig P, Kahn A, Robine S, Perret C, Romagnolo B: Cryptrestricted proliferation and commitment to the Paneth cell lineage following APC loss in the mouse intestine. Development 2005; 132: 1443-1451.

-9 Ouellette AJ, Greco RM, James M, Frederick D, Naftilan J, Fallon JT: Developmental regulation of cryptdin, a corticostatin/defensin precursor mRNA in mouse small intestinal crypt epithelium. J Cell Biol 1989;108:16871695.

10 Karlsson J, Putsep K, Chu H, Kays RJ, Bevins $\mathrm{CL}$, Andersson M: Regional variations in $\mathrm{Pa}$ neth cell antimicrobial peptide expression along the mouse intestinal tract. BMC Immunol 2008;9:37.

- 11 Putsep K, Axelsson LG, Boman A, Midtvedt T, Normark S, Boman HG, Andersson M: Germ-free and colonized mice generate the same products from enteric prodefensins. J Biol Chem 2000;275:40478-40482.

12 Kobayashi KS, Chamaillard M, Ogura Y, Henegariu O, Inohara N, Nunez G, Flavell RA: NOD2-dependent regulation of innate and adaptive immunity in the intestinal tract. Science 2005;307:731-734.

-13 Schmidt DR, Holmstrom SR, Fon Tacer K, Bookout AL, Kliewer SA, Mangelsdorf DJ: Regulation of bile acid synthesis by fat-soluble vitamins A and D. J Biol Chem 2010;285: 14486-14494.

14 Pfaffl MW: A new mathematical model for relative quantification in real-time RT-PCR. Nucleic Acids Res 2001;29:e45.

-15 Edwards U, Rogall T, Blocker H, Emde M, Bottger EC: Isolation and direct complete nucleotide determination of entire genes. Characterization of a gene coding for $16 \mathrm{~S}$ ribosomal RNA. Nucleic Acids Res 1989;17: 7843-7853.

16 Muyzer G, de Waal EC, Uitterlinden AG: Profiling of complex microbial populations by denaturing gradient gel electrophoresis analysis of polymerase chain reaction-amplified genes coding for 16S rRNA. Appl Environ Microbiol 1993;59:695-700.
7 Wang Q, Garrity GM, Tiedje JM, Cole JR: Naive bayesian classifier for rapid assignment of rRNA sequences into the new bacterial taxonomy. Appl Environ Microbiol 2007;73:5261-5267.

18 Brandl K, Plitas G, Mihu CN, Ubeda C, Jia T, Fleisher M, Schnabl B, DeMatteo RP, Pamer EG: Vancomycin-resistant enterococci exploit antibiotic-induced innate immune deficits. Nature 2008;455:804-807.

19 Rupnik M, Wilcox MH, Gerding DN: Clostridium difficile infection: New developments in epidemiology and pathogenesis. Nat Rev Microbiol 2009;7:526-536.

20 Willing BP, Russell SL, Finlay BB: Shifting the balance: antibiotic effects on host-microbiota mutualism. Nat Rev Microbiol 2011;9: 233-243.

21 Lopez-Boado YS, Wilson CL, Hooper LV, Gordon JI, Hultgren SJ, Parks WC: Bacterial exposure induces and activates matrilysin in mucosal epithelial cells. J Cell Biol 2000;148: 1305-1315.

-22 Wehkamp J, Harder J, Weichenthal M, Schwab M, Schaffeler E, Schlee M, Herrlinger KR, Stallmach A, Noack F, Fritz P, Schroder JM, Bevins CL, Fellermann K, Stange EF: NOD2 (CARD15) mutations in Crohn's disease are associated with diminished mucosal alpha-defensin expression. Gut 2004;53:1658-1664.

23 Wehkamp J, Salzman NH, Porter E, Nuding S, Weichenthal M, Petras RE, Shen B, Schaeffeler E, Schwab M, Linzmeier R, Feathers RW, Chu H, Lima H Jr, Fellermann K, Ganz T, Stange EF, Bevins CL: Reduced paneth cell alpha-defensins in ileal Crohn's disease. Proc Natl Acad Sci USA 2005;102:18129-18134.

24 Bevins CL, Salzman NH: Paneth cells, antimicrobial peptides and maintenance of intestinal homeostasis. Nat Rev Microbiol 2011;9:356-368.

25 Giaffer MH, Holdsworth CD, Duerden BI: The assessment of faecal flora in patients with inflammatory bowel disease by a simplified bacteriological technique. J Med Microbiol 1991;35:238-243.

-26 Willing BP, Dicksved J, Halfvarson J, Andersson AF, Lucio M, Zheng Z, Jarnerot G, Tysk C, Jansson JK, Engstrand L: A pyrosequencing study in twins shows that gastrointestinal microbial profiles vary with inflammatory bowel disease phenotypes. Gastroenterology 2010;139:1844-1854.
27 Lin PW, Simon PO, Jr., Gewirtz AT, Neish AS, Ouellette AJ, Madara JL, Lencer WI: Paneth cell cryptdins act in vitro as apical paracrine regulators of the innate inflammatory response. J Biol Chem 2004;279:1990219907.

28 Shi J, Aono S, Lu W, Ouellette AJ, Hu X, Ji Y, Wang L, Lenz S, van Ginkel FW, Liles M, Dykstra C, Morrison EE, Elson CO: A novel role for defensins in intestinal homeostasis: regulation of Il-1beta secretion. J Immunol 2007;179:1245-1253.

29 Giesemann T, Guttenberg G, Aktories K: Human alpha-defensins inhibit clostridium difficile toxin B. Gastroenterology 2008; 134 : 2049-2058.

30 Dessein R, Gironella M, Vignal C, PeyrinBiroulet L, Sokol H, Secher T, Lacas-Gervais S, Gratadoux JJ, Lafont F, Dagorn JC, Ryffel B, Akira S, Langella P, Nunez G, Sirard JC, Iovanna J, Simonet M, Chamaillard M: Tolllike receptor 2 is critical for induction of Reg3 beta expression and intestinal clearance of Yersinia pseudotuberculosis. Gut 2009;58:771-776.

31 Gong J, Xu J, Zhu W, Gao X, Li N, Li J: Epithelial-specific blockade of MyD88-dependent pathway causes spontaneous small intestinal inflammation. Clin Immunol 2010; 136:245-256.

32 Vaishnava S, Behrendt CL, Ismail AS, Eckmann L, Hooper LV: Paneth cells directly sense gut commensals and maintain homeostasis at the intestinal host-microbial interface. Proc Natl Acad Sci USA 2008; 105: 20858-20863.

33 Hoffmann A, Leung TH, Baltimore D: Genetic analysis of NF-kappab/Rel transcription factors defines functional specificities. EMBO J 2003;22:5530-5539.

34 Shirafuji Y, Tanabe H, Satchell DP, Henschen-Edman A, Wilson CL, Ouellette AJ: Structural determinants of procryptdin recognition and cleavage by matrix metalloproteinase-7. J Biol Chem 2003;278:7910-7919. 